\title{
MEJORES PRÁCTICAS PARA IMPLANTAR EL GOBIERNO DE TECNOLOGÍAS DE LA INFORMACIÓN (TI), EN LA UNIVERSIDAD ECUATORIANA
}

\author{
BEST PRACTICES FOR IMPLEMENTING THE INFORMATION TECNOLOGY (IT) \\ GOVERNANCE IN THE ECUADORIAN UNIVERSITY
}

\author{
Diego Marcelo Cordero Guzmán ${ }^{1}$ \\ Unidad Académica de Ingeniería de Sistemas, Eléctrica y Electrónica \\ ${ }^{1}$ Universidad Católica de Cuenca (UCACUE) \\ Cuenca-Ecuador \\ dcordero@ucacue.edu.ec ${ }^{1}$
}

\begin{abstract}
Resumen
El presente artículo contempla un análisis del marco teórico disponible sobre Gobierno de Tecnologías de la Información (TI), que es de aplicabilidad en varios sectores de la industria, el comercio la banca, los servicios, en diferentes zonas y latitudes; se referencia al estado de la universidad en el Ecuador y se insiste que en este contexto es todavía en donde existe mucho que indagar e investigar en el medio, de modo que basados en estos resultados se propongan modelos, métodos, mecanismos, mejores prácticas, que vuelvan eficiente a la Universidad apoyados en las Tecnologías de la Información.

Se inicia con una abstracción del Gobierno Institucional, y su alineamiento con el Gobierno de TI, se describe la literatura sobre marcos de referencia para Gobierno de Tecnologías de la Información, como: ISO/IEC 38500:2008, VAL IT (valor de TI), COBIT (Objetivos de Control para Información y Tecnologías Relacionadas), ITIL (Biblioteca de Infraestructura de Tecnologías de la Información) y conceptos importantes de prácticas relacionadas. Adoptar un modelo que sea conveniente a los intereses de la universidad de la región es la premisa, puesto que la realidad de organismos pertenecientes a otras esferas, es diferente a la nuestra.
\end{abstract}

Palabras Claves: Gobierno, Universidad, Gobierno de TI (Tecnologías de la Información), COBIT, ITIL.

\begin{abstract}
This article provides an analysis of available theoretical framework on Government Information Technology (IT), which is applicable in various sectors of industry, commerce, banking, services in different zones and latitudes; is reference to university status in Ecuador and insists that in this context where there is still much to investigate and research in the middle, so that models based on these results, methods, mechanisms, best practices, proposed that efficient return to the University resting on Information Technology.

It begins with an abstraction of Institutional Government and its alignment with IT governance, the literature on frameworks for Government Information Technology described as: ISO/IEC 38500:2008, VAL IT (TI value), COBIT (Control Objectives for Information and related Technology), ITIL (Infrastructure Library Information Technology) and important concepts related and practical. Adopt a model that is appropriate to the interests of the university in the region is the premise, since the reality of bodies belonging to other areas, is different from ours.
\end{abstract}

Keywords: Government, University, IT (Information Technology) Governance, COBIT, ITIL. 


\section{Introducción}

La universidad es la que despierta las inquietudes del saber, es fuente de la creatividad humana, es el puente entre el pasado y un futuro promisorio. Con su propia autonomía [1] va forjando estados de libertad que tendrán que ser ejecutados con responsabilidad. En su estructura misma, involucra una serie de áreas funcionales dedicadas a la gestión administrativa, financiera, educativa. Con una gran variedad de oferta académica mantiene interacción continua entre estudiantes, docentes, comunidad, personal administrativo y directivo.

La universidad como tal aborda grandes cúmulos de información pasada y activa, de todos los procesos relacionados con su quehacer. Para afrontar esta acuciante necesidad de almacenamiento, proceso y protección de información de todos los estamentos estratégicos, tácticos, operativos, estudiantes, comunidad y demás partes interesadas; la universidad al igual que el resto de organizaciones, ha involucrado el soporte y apoyo de las Tecnologías de la Información (TI, término que será usado a lo largo del presente artículo), junto con el talento humano idóneo, así como las prácticas y procesos para su operación, gestión y gobierno [2], que implican: elementos de hardware; herramientas de software; profesionales técnicos con diversa formación en ramas de la informática

Es preciso cuestionarse si: ¿al interior de este tipo de instituciones se está haciendo uso efectivo de las tecnologías de la información (TI)?. Algunos autores como Sigalés [3], IDC [4], en respuesta a la interrogante planteada, describen que no todas las experiencias de incorporación de las TI a la actividad universitaria han generado los resultados que se habían esperado, pues existen algunos factores claves que están implicados en una adecuada integración de las TI y que responden a una relación con la visión, misión, estrategia y valores de la propia universidad. De forma tradicional las TI han sido usadas como un medio para soportar las operaciones de una organización, antes que los aspectos estratégicos.

En consecuencia, TI debe estar destinada a desempeñar, a más de tareas operativas y tácticas, las de orden estratégico. Para habilitar el cambio Dux Diligens [5], recomienda que TI adicional a soportar a niveles inferiores y medios en la organización, también debe atender los estratégicos. De modo que pase a ser socio, colaborador y facilitador de soluciones clave; la consecución de un buen Gobierno de las TI es fundamental para lograrlas.

Es así que el interés por la implementación de actividades relevantes al Gobierno de TI varía alrededor del mundo, en un comparativo por regiones el rango de preocupación de menor a mayor es el siguiente: Latinoamérica, Asia, Europa, Norteamérica [6]. Esto para la región es crítico, pues hablar de América Latina implica referenciar a un grupo de países heterogéneos y complejos, con grados de desarrollo y experiencias variados, de difícil comparación. A pesar de ello, estas experiencias convergen en vías comunes y comportamientos semejantes, uno de éstos es el aspecto informático [7], presente en la mayoría de organizaciones. Por lo tanto esta similitud también está presente en el desarrollo del Gobierno de TI.

Ángel Peña Ibarra, indica el gran potencial que se tiene en la región para implementar prácticas de Gobierno de TI, ya sea en base a marcos de referencia existentes o con la generación de nuevos modelos que se adapten a la naturaleza, madurez e idiosincrasia de las organizaciones [8].

Uno de los ejes estratégicos críticos de la universidad es "la calidad", excluir a las TI del proceso de generación de estas estrategias no sería factible, puesto que se han constituido en un instrumento importante para agregar calidad [9]. De hecho el entorno que se cierne sobre la universidad ecuatoriana está marcado desde el Gobierno Central, con el proceso de impulso a la calidad de la Educación Superior emanado desde la reforma constitucional del 2008 y viabilizado a través de una nueva Ley Orgánica de Educación Superior. Los cambios y transformaciones internas de las universidades son cada vez más intensos, pues están orientados a cumplir con los indicadores de los modelos de acreditación impuestos por los organismos de control de la calidad en la educación. Un eficiente soporte de la informática es requerido para proceso y entrega de información con parámetros de calidad, por ello el Gobierno de TI se ha convertido en un factor crítico.

Para Cornella [10] es de suma prioridad para la organización mejorar y evaluar la calidad de su información, puesto que la Institución como tal es información y la calidad de ésta es un fiel reflejo de la administración [11]. En las organizaciones prestadoras de servicio es importante conocer el nivel de satisfacción de sus partes interesadas, traducido en información sobre sus expectativas y percepciones [12]. Dado que la educación es un servicio, le corresponde a la universidad gestionar calidad en la información que procesa, es así que existen varios estudios en donde se destaca la evaluación y mejora de calidad en la información [13], [14], [15].

El artículo abarca un análisis del marco teórico disponible sobre Gobierno de TI, que es de aplicabilidad en varios sectores de la industria, el comercio la banca, los servicios, en diferentes zonas y latitudes. Referencia al estado de este Gobierno en la universidad, y se insiste, que en este contexto es 
todavía en donde existe mucho que indagar, e investigar, de modo que basados en estos resultados, se propongan modelos, formas, métodos mecanismos, mejores prácticas, que vuelvan eficiente la Gestión de TI, en la universidad.

Modelos de esta naturaleza serán convenientes a los intereses organizacionales de la región, puesto que la realidad de los Gobiernos de TI al interior de organismos pertenecientes a otras esferas, es diferente a la nuestra. Hay factores medioambientales, culturales, económicos, de madurez, sociales que nos diferencian, los cuales inciden en el comportamiento organizacional.

\section{Texto}

Es imprescindible redundar en que es necesario, disponer de un propio Modelo de Gobierno de TI para la Universidad, que rescate o retome aspectos positivos de otras experiencias, mejores prácticas, marcos de referencia para Gobierno de TI, como: ISO/IEC 38500:2008 (estándar para Gobierno de TI), VAL IT (valor de TI, se ocupa de los presupuestos, los costos, riesgos y resultados relacionados con las inversiones de TI [16]), COBIT (siglas en inglés para referirse al término Objetivos de Control para Información y Tecnologías Relacionadas, concebido como un conjunto de mejores prácticas para el Gobierno de TI, [17]), ITIL (siglas en inglés para referirse al término Biblioteca de Infraestructura de Tecnologías de Información, es el enfoque de mayor aceptación, para la gestión de servicios de TI, [18]) y procesos importantes de mejores prácticas relacionadas como los riesgos de TI, que es un marco de trabajo, que brinda una vista completa de todos los riesgos relacionados con el uso de TI [16].

De la juntura es recomendable tomar procesos críticos, es decir los más relevantes y recomendar los niveles de madurez a ser alcanzados. Para generar el modelo, es preciso que se aborden componentes teóricos, como: "Gobierno Institucional”; “Gobierno de TI (GTI)”; “Alineación Estratégica”; "Marcos de Trabajo Relacionados”; "Modelos de Madurez”; “Gestión Universitaria”.

\section{Marco Referencial}

Hay referencias de estudios efectuados en países como España, en donde la Conferencia de Rectores de Universidades Españolas (CRUE), ha planteado con anterioridad el abordar procesos de implantación de sistemas de Gobierno de las TI. Por ello, en el 2009 encargó a un grupo de expertos e investigadores, diseñar un Modelo de Gobierno de las TI específico para Universidades (GTI4U) españolas, que sirva como referencia y facilite la implantación y maduración de sistemas de Gobierno de las TI en las universidades españolas.

En mencionado estudio se afirma que actualmente, los sistemas de Gobierno de las TI se encuentran implantados con éxito en otros sectores (banca, seguros, industria, etc.) alcanzando una madurez de 2,67 sobre 5 en la escala propuesta por el IT Governance Institute (ITGI). En adición hay que recalcar, que también se están incorporando al Gobierno de las TI universidades de todo el mundo, pues según el estudio realizado por Yanosky y Borreson [19] ya alcanzan una madurez de 2,30 sobre 5 , lo que significa que las universidades se encuentran todavía en una situación incipiente y en proceso de maduración.

No se tiene referencia de un estudio serio y detallado que se haya efectuado sobre el Gobierno de TI en la Universidad ecuatoriana, en consecuencia se presume que no se dispone de un marco de referencia fundamentado y científicamente validado, para este sector. Lo que las instituciones educativas han efectuado es implementar sus propias prácticas de gobernanza de TI o asumir en parte los marcos existentes como los mencionados.

\subsection{Bases Teóricas y Conceptuales}

Para disponer del hilo conductor que encamine el conocimiento necesario y entendible, que sustente la base teórica del Gobierno de Tecnologías de la Información en un entorno universitario, es necesario que se parta de la explotación de los conceptos de las Ciencias de la Administración que se van entretejiendo con el enfoque desde arriba hacia abajo (también conocido como top-down) en donde se arranca desde la visión más general hacia la específica, técnica usada en varios campos de aplicación [20], para concatenar el Gobierno Organizacional el de TI y sus habilitadores, como se ilustra en la figura 1.

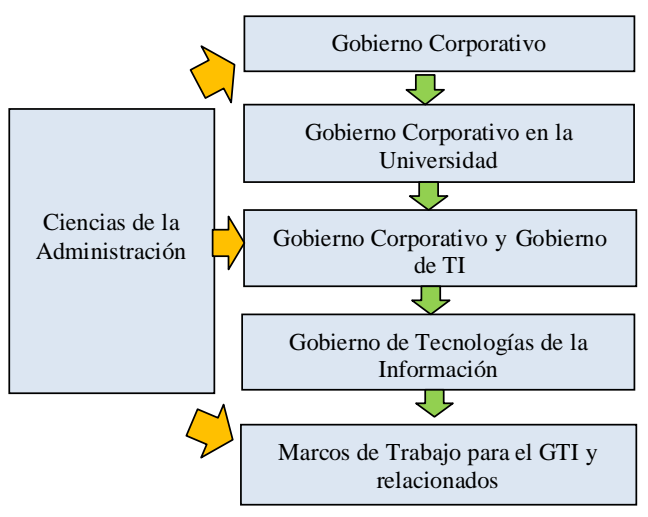

Figura 1. Concatenación del marco teórico para Gobierno de TI en la universidad. Fuente: elaboración propia 
3.1.1 Gobierno Institucional, Gobierno de TI y Alineación Estratégica

De hecho en las instituciones alrededor del mundo, está latente, cada vez más la preocupación por administrarlas adecuadamente. Entre el rango de áreas de interés, se encuentra la gestión de un buen Gobierno Corporativo, con la intención de generar confianza en el mercado, proporcionando información financiera transparente. En este contexto, "el gobierno corporativo se convierte en un conjunto de reglas que guían y limitan la acción de los gerentes y establecen los fundamentos de la gobernanza para las organizaciones” [21].

El Programa de Buen Gobierno Corporativo (BGC) desarrollado en Ecuador por la Bolsa de Valores de Quito, tiene como objetivo contribuir al desarrollo de la oferta y demanda de prácticas de gobierno corporativo en empresas ecuatorianas y aumentar la capacidad local de implementar dichas prácticas [22]. Con este escenario previo, y el apoyo de instituciones públicas y privadas, se da la creación del Instituto Ecuatoriano de Gobernanza Corporativa (IEGC), en cuya misión se enmarca la inminente prioridad de ser el referente para el Ecuador en promoción, difusión, soporte y apoyo a emprendimientos, implementaciones, estudios, investigaciones, sobre buenas prácticas de GC en las empresas [23].

El Ecuador luego de haber soportado un período de inestabilidad política ${ }^{1}$, con la destitución de varios gobernantes, a manos de grupos de poder con intereses particulares, decide reafirmar un cambio en las urnas en el proceso eleccionario presidencial del 2006, y apuesta por una nueva propuesta de gobierno, que proyecta un proceso de cambio en el modelo de desarrollo del país.

Para ello era necesario reformar políticamente al estado ecuatoriano; el camino se abre, con la ratificación de cambio a través de consulta popular, que apoya la formación de una renovada Asamblea Constituyente, encargada de redactar una nueva Constitución de la República, puesta en vigencia en octubre de 2008, que entre sus bases doctrinarias, propone establecer una nueva forma de convivencia ciudadana, contemplando la diversidad, el ambiente armónico con la naturaleza y el entorno, para alcanzar el buen vivir, el sumak kawsay ${ }^{2}$. En los

\footnotetext{
1 El Ecuador desde 1996 hasta el 2007, fue gobernado por 11 presidentes, dando lugar al período de mayor inestabilidad gubernamental en su historia.

2 O Suma Qamaña, en aimara (lengua de algunos pueblos indígena de Bolivia, Perú, Chile, Argentina) forma parte del discurso político actual de los gobiernos de Ecuador y Bolivia. Este término ha sido traducido como "Buen Vivir", cuya acepción más pertinente sería "Vida en Plenitud", doctrina, retomada y recreada de las vivencias ancestrales de los pueblos indígenas; las
}

artículos de la Constitución del 2008, que están referenciados al sistema nacional de educación, se prevé la necesidad de dictar una nueva Ley Orgánica de Educación Superior (LOES), coherente con los nuevos principios constitucionales establecidos en la Carta Suprema, vigente desde el 2008.

La LOES, creada por la Asamblea Nacional [24] contempla entre sus doctrinas, el "principio de calidad”, que consiste en la búsqueda continua de la excelencia académica; éste, se encadena con la "evaluación de la calidad”, para determinar las condiciones de la institución, con la intención de generar reformas y mejoras en los programas de estudios, las carreras o la misma institución como tal, es un proceso constante e implica seguimiento continuo.

Con este marco regulatorio aprobado y en vigencia desde el 2008, las universidades del Ecuador han estado sujetas, a una serie de cumplimientos, entrega de evidencia, cambios internos para generar mejora continua, evaluaciones, mejoras de procesos, reingeniería de personal, archivo de información, proceso de datos, etc. En este lapso de tiempo la dependencia hacia las tecnologías de la información se ha incrementado, y es necesario disponer de un adecuado gobierno, para que éstas apoyen la estrategia institucional.

Es válido afirmar que las organizaciones e instituciones dependen cada vez más de las tecnologías de información, para su operatividad y desarrollo. Por tanto éstas ejecutan grandes y significativas inversiones en TI, todo con el objetivo de alcanzar la eficiencia, mayor seguridad, apoyo a la consecución de la misión y visión, concordante con los objetivos del plan estratégico. Sin embargo en una gran mayoría, las áreas estructurales y funcionales, no tienen adecuados niveles de comunicación, los esfuerzos de un área son desconocidos por otras; una de éstas es el área de TI, que en muchas ocasiones tiene sus objetivos definidos, pero éstos no están necesariamente concordantes con los de la organización. Con frecuencia existe una pobre alineación estratégica entre ambos, debido a que los ritmos de desarrollo entre TI y la organización son diferentes [25].

De acuerdo a Weill, Subramani, y Broadbent [26], el área de TI soporta diferentes presiones y compromisos desde las unidades usuarias, pues debe apoyar la operatividad de la organización, y dar respuesta a aspectos regulatorios, técnicos y de comercio. El esfuerzo invertido en cumplir estas demandas, puede hacer que fácilmente el área de TI

mismas que al ser recuperadas se transforman en formas políticas de resistencia al capitalismo y a las alternativas a ese mismo sistema. 
pierda el alineamiento estratégico con la organización, y se dedique más bien, a resolver tareas puntuales y problemas técnicos concretos.

Determinar el concepto de Gobierno de TI (GTI), y todas sus implicaciones, de modo que se logre la alineación e integración con el G.C., ha sido y es, un constante esfuerzo de organizaciones de estandarización, instituciones de regulación, consultoras, la academia y centros de investigación.

Según ITGI [27], Gobierno de TI es la responsabilidad de los ejecutivos y junta directiva. Consiste en el liderazgo, estructura organizacional, y procesos, que aseguran que TI soporta la estrategia y objetivos del negocio.

Para Van Grembergen [28], Gobierno de TI es la capacidad de organización ejercida por el nivel directivo, ejecutivos y gerentes de TI, para controlar la formulación e implementación de estrategias de TI, de modo que se asegure la integración entre TI y la organización.

Tomando en cuenta que la participación de la organización es crucial, Grembergen y De Haes [29], definen que el Gobierno Empresarial de TI es parte del Gobierno Corporativo, y comprende la determinación e implementación de procesos, estructuras y mecanismos relacionales, que habilitan al talento humano tanto de la organización como de TI, para ejecutar sus responsabilidades, de modo que puedan agregar valor, a las inversiones de TI.

El Gobierno de TI integra e institucionaliza las buenas prácticas, de modo que TI soporta los objetivos organizacionales. Permite a la organización aprovechar al máximo la información, maximizar los beneficios, capitalizar las oportunidades y generar ventajas competitivas [30]. Para otros autores, se puede definir como el uso eficiente de los recursos de TI para apoyar el cumplimiento de los objetivos de la organización [31]; o el gobierno abierto, que incorpora como pilares fundamentales de su funcionamiento: la transparencia, participación y colaboración con la ciudadanía, en función de las oportunidades de las tecnologías de la información y la comunicación [32].

De acuerdo a Weill y Ross [33], el Gobierno de Empresa tiene dos dimensiones: el Gobierno Corporativo y el Gobierno de Negocio, las cuales deben estar equilibrados como los péndulos de una balanza; cada dimensión se mueve por su propia misión: la conformidad y desempeño en cada caso. La conformidad tiene que ver con la legislación, políticas y requerimientos de auditoría; en cambio el desempeño, exige que las tecnologías de información se involucren en la organización, esto se ilustra en la figura 2 .

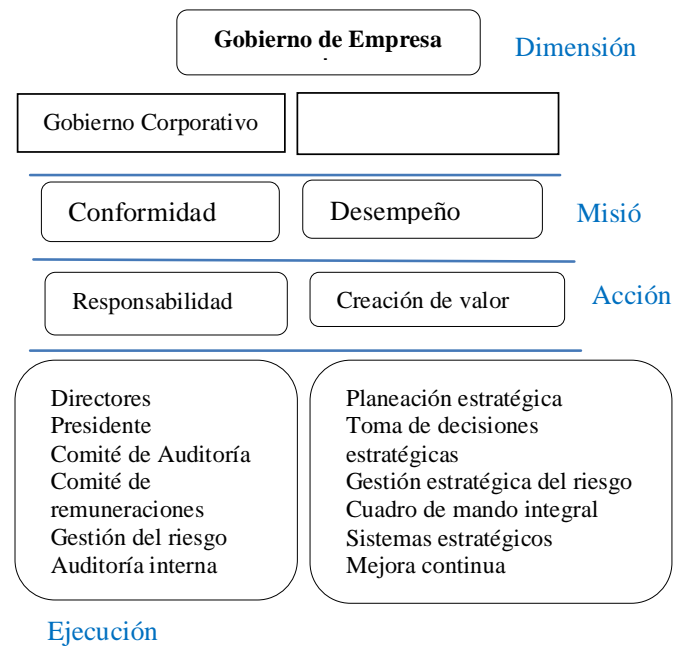

Figura 2. Gobierno de Empresa con sus dimensiones Fuente: elaboración en base al modelo Peter Weill y Jeanne W. Ross [33]

El Gobierno de TI, implica alineación de la tecnología de información con la institución. El alineamiento Institución Vs TI, se constituye en un factor importante, para entregar valor a la organización a través de la inversiones de TI, como se indica la figura 3 [34].

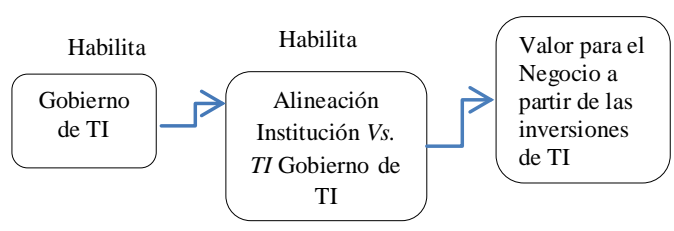

Figura 3. Alineación de TI, con el negocio para entrega de valor. Fuente: elaborado y adaptado de [34]

Henderson y Venkatraman [35], fueron los primeros en describir de manera clara la relación entre estrategias del negocio y estrategias de TI, a través del Modelo de Alineamiento Estratégico, conocido como modelo SAM (Strategic Alignment Model). Este se basa en cuatro perspectivas (estrategia del negocio, estrategia de TI, infraestructura y procesos organizacionales, infraestructura y procesos de TI), que están distribuidas en dos dimensiones (ajuste estratégico, integración funcional), que en su conjunto forman la estrategia global de la organización, como se indica en la figura 4.

Ajuste estratégico: clasifica los dominios de alineamiento en externo e interno. El dominio externo se refiere a la posición de una organización relativa a sus competidores. El dominio interno se refiere a la estructura de la organización. 


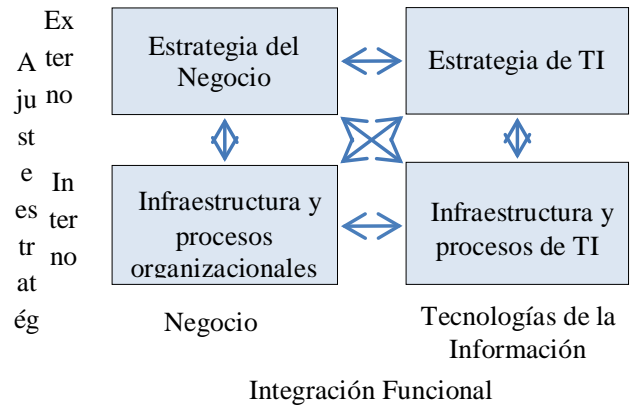

Figura 4. Modelo SAM. Fuente: elaboración en base a la modelo de Henderson y Venkatraman [35]

Integración funcional: Se distinguen dos tipos la integración estratégica y la operacional. La estratégica es el enlace entre la estrategia del negocio y la estrategia de TI, buscando generar al negocio ventaja estratégica competitiva. La operacional cubre el enlace entre, la infraestructura y procesos organizacionales, y los de TI [36].

\section{Mejores Prácticas para Gobierno de TI}

A continuación se detalla lo relevante de los marcos de referencia: ISO 38500:2008, VAL IT, COBIT, ITIL, considerados por el autor como los de mayor criticidad para implementar el Gobierno de $\mathrm{TI}$, al interior de la universidad.

\subsection{ISO/IEC 38500:2008}

Por la importancia que tiene el Gobierno de TI, la Organización Internacional para la Estandarización (ISO), liberó en el año 2008, un nuevo estándar, denominado: "Gobierno Corporativo de TI" (ISO $^{3} /$ IEC $^{4}$ 38500:2008). Norma que permite identificar el papel fundamental que representan los consejos y miembros de la alta dirección de las organizaciones [37], así como como su campo de alcance, que se extiende al gobierno de los procesos de TI de todo tipo de organización. Para Fernández [38], la dirección debe gobernar las TI mediante 3 tareas principales: evaluar, dirigir, monitorizar. Por otro lado, para Martínez [39], la norma ISO/IEC 38500:2008, establece, a nivel internacional, una serie de directrices, básicas o generalistas, de orientación a la alta dirección en relación con el buen gobierno corporativo de TI. La norma alienta a utilizar seis dimensiones para que la organización pueda obtener sus objetivos de TI. Estos se traducen en:
- Responsabilidad. Todos comprenden y aceptan sus responsabilidades en la oferta o demanda de TI

- Estrategia. Toma en cuenta capacidades actuales y futuras de TI, que provienen de la estrategia de la organización.

- La adquisición de TI. Se ejecuta de manera técnica con equilibrio entre beneficios, oportunidades, costos, riesgos, en el corto y mediano plazo.

- Rendimiento. TI da soporte a la organización, a través de servicios con la calidad.

- Conformidad. La función de TI cumple leyes y normas.

- Factor humano. Las prácticas y decisiones demuestran respeto al factor humano.

\subsection{VAL IT}

En primera instancia para Garzás y Cabrero [40], es recomendable contar con una clara definición de los términos, descritos en este contexto, así:

- Valor. Es el resultado final de la organización después de una inversión en TI. Este resultado pueden ser económico, no económico o una combinación de los dos.

- Portafolio. Es el conjunto de programas, proyectos, recursos, servicios.

- Programa. Grupo estructurado de proyectos necesarios y suficientes para lograr resultado comercial y entregar valor.

- Proyecto. Grupo de actividades estructuradas y relacionadas entre sí, para entregar una capacidad definida, en base a un plan y recursos acordados.

Para el Governance Institute [41], VAL IT, es el conjunto de las mejores prácticas que permiten medir, monitorizar y optimizar la realización de valor para el negocio, a partir de la inversión en TI. Estas inversiones pueden generar beneficios, cuando estén disponibles procesos de gobierno apropiados, junto con el compromiso y participación del nivel directivo organizacional. Pues según McFarlan y Richard [42], la falta de control por parte de las autoridades de la organización sobre las actividades de TI es peligrosa; se correría un riesgo semejante, al que se produciría, si se dejaran de auditar las cuentas contables.

Las inversiones de la organización posibilitadas por TI, tendrían un nivel conocido y aceptable de riesgo, y con ellas lo pertinente es implementar el cambio. Es indispensable aclarar, que el valor de la organización se da en función de lo que se hace con TI, más no la tecnología en sí.

\subsection{COBIT}

\footnotetext{
${ }^{3}$ Organización Internacional de Estandarización.

${ }^{4}$ Comisión Electrotécnica Internacional.
} 
Es el modelo para el Gobierno de Tecnologías de la Información (TI), desarrollado por la Information Systems Audit and Control Association (ISACA) y el IT Governance Institute (ITGI). A continuación se lista una serie de características de COBIT [43] :

- Proporciona buenas prácticas que representan el consenso de los expertos.

- Las prácticas están enfocadas de manera central en el control, antes que en la ejecución.

- Las prácticas prevén optimizar las inversiones facilitadas por TI, asegurar la entrega del servicio de TI y tener un mecanismo de control sobre lo que hace $\mathrm{TI}$.

- Mantiene orientación al negocio, vincula las metas del negocio con las metas de TI.

- Proporciona métricas y modelos de madurez para medir capacidad de los procesos.

- Identifica responsabilidades de los propietarios de los procesos de negocio y de TI.

- Tiene un modelo de procesos que representa los que normalmente se encuentran en las funciones de TI.

- Los procesos, pertenecen a cincos dominios: Alineación Estratégica de la Institución con Tecnologías de la Información, Administración de Recursos de TI , Administración de Riesgos de TI, Administración del Rendimiento de TI, Entrega de valor de TI.

Para Pradini y Szuster [44], Cobit 5 incorpora los pensamientos más recientes sobre gobierno empresarial y técnicas de administración. Busca la integración de otros importantes marcos como las normas ISO, Risk IT (riesgos de TI), Val IT, ITIL; a la vez que es un marco de trabajo que distingue y separa al gobierno de la gestión de TI. Para [45], los 5 principios de COBIT 5, se contemplan en: satisfacer las necesidades de las partes interesadas; cubrir la organización en forma integral; aplicar un solo marco integrado; habilitar un enfoque holístico.

Los modelos de Madurez, en COBIT como se indica en la figura 5 , responden a tres necesidades: una medición relativa de dónde se encuentra la empresa, una manera de decidir hacia dónde ir de forma eficiente, una herramienta para medir el avance contra la meta.

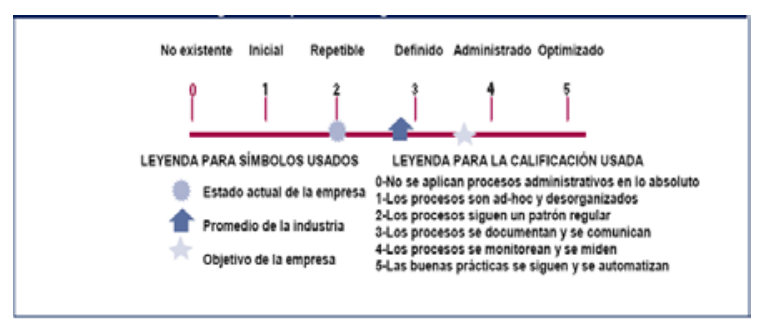

Figura 5. Modelos de Madurez según COBIT. Fuente: Governance Institute [41]

COBIT se compone de 5 dominios: Planear y Organizar (APO), Adquirir e Implementar (BA), Entregar y dar servicio (DS) y Monitorear y Evaluar (ME), Gobierno de TI (EDM), organizados en 37 procesos, como se indica en la figura 6 , que buscan cumplir las necesidades de información de la organización en cuanto a: efectividad, eficiencia, confidencialidad, integridad, disponibilidad, cumplimiento, confiabilidad, a través del uso eficiente de los recursos de TI: aplicaciones, información, infraestructura y personas.

\subsection{ITIL}

Para el ITSMF (Fórum para la Gestión de Servicios Informáticos) se ha convertido en el estándar mundial en la Gestión de Servicios Informáticos. El conjunto de mejores prácticas ITIL, provee un conjunto completo de recomendaciones que abarca no sólo los procesos y requerimientos técnicos y operacionales, sino que se relaciona con la gestión estratégica, la gestión de operaciones y la gestión financiera de una organización moderna.

El tema de Gestión de Servicios (Soporte al Servicio y Provisión al Servicio), como se representa en la figura 7, es el más ampliamente difundido e implementado. El conjunto de mejores prácticas ITIL, provee un conjunto completo de recomendaciones que abarca no sólo los procesos y requerimientos técnicos y operacionales, sino que se relaciona con la gestión estratégica, la gestión de operaciones y la gestión financiera de una organización moderna.

Para ITSMF, "Soporte al Servicio", contempla los procesos que garanticen la continuidad, disponibilidad y calidad del servicio prestado al usuario y comprende: mesa de ayuda; base de conocimientos; gestión de: incidentes, problemas, cambios y versiones. Por otro lado "Provisión del Servicio”, se ocupa de los procesos: administración de la configuración; gestión: del nivel de servicio, de la disponibilidad, de la capacidad, de la viabilidad financiera, de continuidad.

\section{Conclusiones}

Disponibles están una variedad de marcos de referencia, mejores prácticas, como las abordadas en el artículo, y su aplicación ha sido ya validada en empresas de diferente índole, a excepción de centros de Educación Superior, por las pocas o escasas referencias disponibles.

Es preciso que se efectúen investigaciones, serias y profundas sobre las realidad del medio y del país 
sobre el Gobierno de TI en la universidad, y se propongan modelos particularizados para este sector

En la universidad ecuatoriana, debe incentivarse a una proliferación de cultura informática, que permita concienciar la necesidad de disponer de un Gobierno de TI, que genere madurez en los procesos de TI.

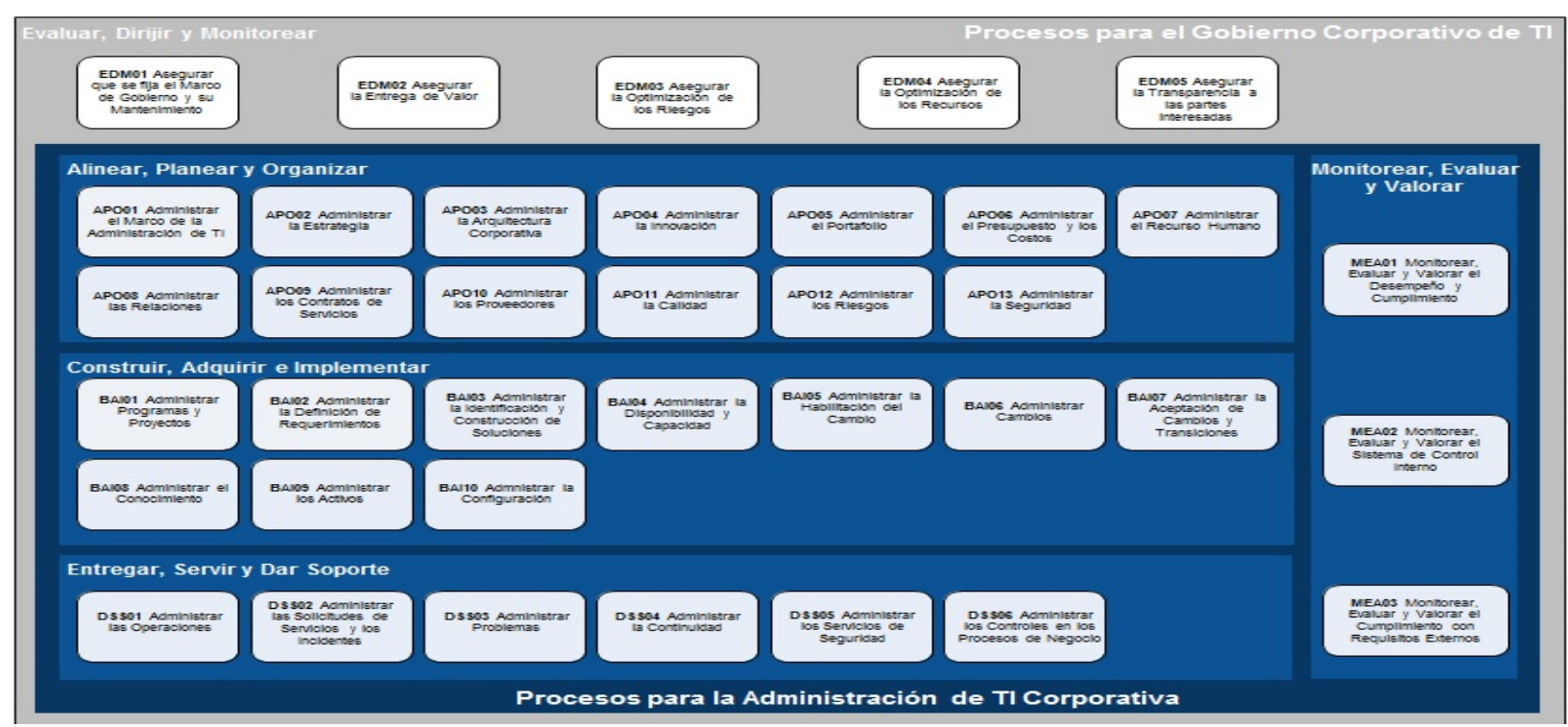

Figura 6. Alineación de TI, con el negocio para entrega de valor.

Fuente: [46]

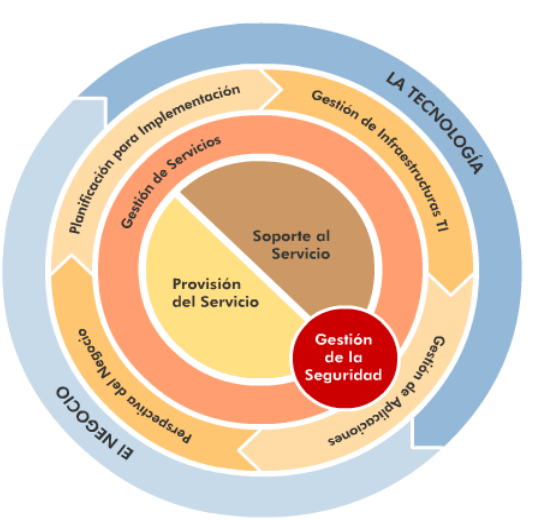

Figura 7. Marco de Trabajo General de ITIL. Fuente: http:/litil.osiatis.es/Curso_ITIL/Gestion_Servicios_TI/fun damentos_de_la_gestion_TI

Es bien sabido que el presupuesto destinado a la educación, en la región siempre ha estado en contraposición, a las demandas reales de desarrollo de los pueblos, esto ha implicado que las universidades a pesar de las limitaciones impuestas por factores externos, y a costa de su propio esfuerzo y creatividad, busquen efectividad en su gestión. La misma puede ser fortalecida por la eficiencia de las operaciones internas, procesos y recursos, apoyada por las tecnologías de la información, producto de aplicar modelo efectivos sobre Gobierno de TI.
Un modelo de Gobierno de TI, para la universidad, tendrá componentes estratégicos, tácticos y operativos. La parte estratégica estaría fundamentada por el alineamiento al Plan Estratégico, la participación del nivel Directivo en las decisiones de TI; el aspecto táctico por el cumplimiento de regulaciones, la optimización del costo, la eficiencia de soporte a los requerimientos de información de la Institución; y el aspecto operativo soportado por los procesos de gobierno de TI

Lo plasmado en el artículo dará apertura, para que se efectúen desde el campo académico temas investigativos sobre Gobierno de TI, sobre todo en el sector educativo del país.

\section{Agradecimientos}

A la Unidad Académica de Ingeniería de Sistemas, Eléctrica y Electrónica, de la Universidad Católica de Cuenca, por la apertura a procesos de orden investigativo sobre aspectos del Gobierno de TI.

\section{Referencias}

[1] S. Gangotena, «Qué es la Universidad,» El Comercio, p. 2A, 11 Mayo 1999.

[2] J. Cabero, «Las Nuevas Tecnologías en la 
Actividad Universitaria,» Revista de Medios y Educación, pp. 80-100, 2003.

[3] C. Sigalés, «Formación universitaria y TIC: nuevos usos y nuevos roles,» Revista de Universidad y Sociedad del Conocimiento, vol. 1, pp. 1-2, Septiembre 2004.

[4] IDC, TIC para PYMES, España: LID Editorial Empresarial, 2007, pp. 50-52.

[5] Dux Diligens, «Gobierno y Gestión Estratégica,» 2010. [En línea]. Available: http://www.duxdiligens.com/gobierno-gestionestrategica-ti.shtml. [Último acceso: 15 Mayo 2014].

[6] R. Meadows, «Revela Encuesta Los Principales Problemas de Gobierno de Las TI,» 13 Febrero 2008. [En línea]. Available: http://www.isaca.org/About-ISACA/Pressroom/News-Releases/Spanish/Pages/RevelaEncuesta-Los-Principales-Problemas-deGobierno-de-Las-TI.aspx. [Último acceso: 30 04 2014].

[7] C. Batista, «Las TIC para la Gobernabilidad: La contribución de las Tecnologías de la Información y la Comunicación a la gobernabilidad local en América Latina,» Núcleo de Investigación en Políticas Públicas, Brasilia, 2003.

[8] IT Governance Institute, «Los Principales Problemas de Gobierno de las TI,» 15 Febrero 2008. [En línea]. Available: http://www.compuchannel.net/2008/02/15/it/. [Último acceso: 25 Abril 2014].

[9] A. Pedreño, «La Cooperación y las TIC's para la Mejora de la Calidad de la Universidad,» de La Transformación de Las Universidades a través de las TIC: Discursos y Prácticas, Barcelona, UOC, 2004, p. 53.

[10] A. Cornella, Infonomia: la empresa es Información, Madrid: Deusto, 2000, p. 337.

[11] Y. Xiuxa y S. Zhongwen, «A Study on Information Quality Maturity Model in Enterprise,» de Service Operations and Logistics, and Informatics, 2007. SOLI 2007. IEEE International Conference, Philadelphia, 2007.

[12] M. Torres, E. Torres, D. Rojas y C. Vásquez, «Método de evaluación de la calidad de la información en servicios basada en tratamiento borroso,» Unversidad, Ciencia y Tecnología, vol. 12, nº 47, pp. 73-80, 2008.

[13] D. Rojas y M. Torres , «Diseño de un plan para mejorar la calidad de la información en un instituto de previsión social,» UNEXPO, Venezuela, 2004.

[14] M. Andrés, «Evaluación del efecto de la información en la satisfacción de los pacientes atendidos en los servicios de urgencias hospitalarios,» Revista de Calidad Asistencial, pp. 161-167, 2007.

[15] E. Herrera, G. Passi, A. López y C. Porcel, «Evaluating the information quality of Web sites: A methodology based on fuzzy computing with words: Special Topic Section on Soft Approaches to Information Retrieval and Information Access on the Web,» Journal of the American. Society for Information Science and Technology, vol. 57, n 4, p. 538 - 549, 2006.

[16] ISACA, «Val IT Framework for Business Technology Management,» 2013. [En línea]. Available: http://www.isaca.org/KnowledgeCenter/Val-IT-IT-Value-Delivery-/Pages/ValIT1.aspx. [Último acceso: 5 Abril 2013].

[17] Technology TC, «Soluciones Empresariales,» 2013. [En línea]. Available: http://www.technologytc.com/index.php/cobit. [Último acceso: 15 Enero 2013].

[18] ITIL, «ITIL Home,» 2013. [En línea]. Available: http://www.itil-officialsite.com/. [Último acceso: 20 Julio 2013].

[19] R. Yanosky y J. Borreson, «Process and Politics: IT Governnace in Higher Education,» EDUCAUSE, 2008.

[20] M. McCall, «Investopedia,» 10 Septiembre 2013. [En línea]. Available: http://www.investopedia.com/articles/stocks/06/ topdownapproach.asp. [Último acceso: 15 Octubre 2013].

[21] Lafabra y Carrasco, «La Diversidad en el Código de Buen Gobierno Español y la Práctica Empresarial. Conocimiento, Innovación y Emprendedores,» Pecvnia, p. 3350, 2007.

[22] Instituto de Gobierno Corporativo en América Latina, Gobierno Corporativo en América Latina 2010-2011, Washington: Global Corporate Governance, 2011, p. VII.

[23] Instituto Ecuatoriano de Gobernanza Corporativa, «IEGC,» 2013. [En línea]. Available:

http://www.iegc.ec/index.php?option=com_cont ent\&view=article\&id=77\&Itemid=147. [Último acceso: 8 Noviembre 2013].

[24] Asamblea Nacional, «Ley Orgánica de Educación Superior,» Quito, 2010.

[25] J. Ross y P. Weill, «Six IT Decision Your IT People Shouldn’t Make,» November 2002. [En línea]. Available: http://www.qualified-auditpartners.be/user_files/ITforBoards/GVIT_Harv ard_Business_Review-

Ross_Jeane__Weill_Peter_Six_IT_Decsions_ Your_IT_People_Shouldnt_Make_2002.pdf. [Último acceso: 12 Noviembre 2013].

[26] P. Weill, M. Subramani y M. Broadbent, 
«Building IT Infrastructure for Startegic Agility,» MIT, Masachusets, 2002.

[27] ITGI, «Board Briefing on IT Governance,» 2003. [En línea]. Available: www.itgi.org. [Último acceso: 30 Septiembre 2013].

[28] W. Van Grembergen , «Introduction to the Minitrack : IT Governance and its Mechanisms,» de 35th International Conference on System Sciences, Hawaii, 2002.

[29] W. Grembergen y S. De Haes, Enterprise Governance of Information Technology, New York: Springer, 2009.

[30] M. Palao, Reflexión sobre el Estado del Arte del Buen Gobierno TIC, Bogotá: ISACA, 2010.

[31] ITGI, IT Governance, global status report 2008, Rolling Meadows, 2008.

[32] Ministerio de Industria, Energía y Turismo, «Objetivos, estrategias y actuaciones nacionales e internacionales sobre Gobierno Abierto,» Redes, España, 2013.

[33] P. Weill y J. Ross, IT Governance. How Top Performers Manage IT Decision Rights for Superior Results, Boston: Harvard Business School Press, 2004, pp. 4-7.

[34] W. Grembergen y S. De Haes, Enterprise Governance of Information Technology, New York: Springer, 2009, p. 6.

[35] J. C. Henderson y N. Venkatraman, «Strategic Alignment: Lveraging Information Technology for Transforming Organizations,» IBM Systems Journal, vol. 32, 1993.

[36] N. Vargas y L. Plazaola, «Una representación consolidada del alineamiento estratégico de los negocios y las tecnologías de la información,» Nexo, vol. 20, $\mathrm{n}^{\circ}$ 02, pp. 102-112, 2007.

[37] M. Ballester, «Gobierno de las TIC ISO/IEC 38500,» Journal Online, pp. 1-4, 2010.

[38] C. Fernández , «La Norma ISO/IEC 38500Buen Gobierno de las Tecnologías de la Información,» Normas de Gestión Avanzada 2011, pp. 1-21, 2011.

[39] B. Martínez Cándano, «ISO/IEC 38500 y el Buen Gobierno de las TI,» Julio 2008. [En línea].Available:

http://www.criptored.upm.es/guiateoria/gt_m45 3a.htm. [Último acceso: 12 Noviembre 2012].

[40] J. Garzás y D. Cabrero, «Val IT,» 2007. [En línea].Available:

http://www.kybeleconsulting.com/val-it/. [Último acceso: 17 Abril 2014].

[41] IT Governance Institute, «COBIT 4.1,» SeaQuation BV, United States, 2009.

[42] W. McFarlan y N. Richard, «Information, Technology and the Board of Directors,» Harvard Business Review, EEUU, 2005.
[43] IT Governance Institute, COBIT® 4.1: Framework, Control Objectives, Management Guidelines, Maturity Model., United States of America: ITGI, 2007.

[44] P. Pradini y R. Szuster, «Segurinfo 2012,» ISACA Buenos Aires Chapter, Buenos Aires, 2012.

[45] ISACA, COBIT 5 for Information Security, United States of America, 2012, pp. 27-31.

[46] ISACA, «Un Marco de Negocio para el Gobierno y la Gestión de la TI en la Empresa,» Rolling Meadows, 2012. 